\title{
Conditioned Medium Derived from Salidroside-Pretreated Mesenchymal Stem Cell Culture Ameliorates Mouse Lipopolysaccharide-Induced Cerebral Neuroinflammation- Histological and Immunohistochemical Study
}

\author{
Zeinab M. El Maadawi \\ Department of Histology and Cell Biology, Faculty of Medicine, Cairo University, Cairo, Egypt
}

Background and Objectives: Neuroinflammation is involved in the pathogenesis of neurodegenerative disorders. Conditioned medium (CM) derived from bone marrow mesenchymal stem cells (MSCs) revealed substantial benefits due to its rich content of trophic factors. Salidroside (Sal), extracted from Rhodiola rosea, is known for its anti-inflammatory and neuroprotective effects. This study was designed to investigate the effect of Sal pretreated CM (CM-Sal) derived from bone marrow MSCs in lipopolysaccharide (LPS) induced neuroinflammation.

Material and Methods: Fifty adult male mice were equally divided into 5 groups: Group I (Normal Control), Group II (LPS): single $0.8 \mathrm{mg} / \mathrm{kg}$ LPS intraperitoneally; Group III (LPS-DMEM), Group IV (LPS-CM) and Group V (LPS-CM-Sal): LPS was injected as group II followed, 24 hours later, by intranasal injection of $50 \mu 1$ of filtered serum-free Dulbecco's Modified Eagle's medium (DMEM), CM or CM-Sal, respectively, twice daily for 4 days. Animals were sacrificed at day 6 and paraffin cerebral sections were subjected to Hematoxylin and Eosin staining and immunohistochemistry with caspase 3 (apoptosis), glial fibrillary acidic protein GFAP (astrocytes) and CD68 (active microglia) followed by quantitative morphometric study.

Results: Examination of LPS and LPS-DMEM groups revealed neuronal apoptosis with reactive astrogliosis and increased active microglia. LPS-CM and LPS-CM-Sal groups showed less apoptosis, less astrocytes and less active microglia. The regression in neuroinflammation was more evident in LPS-CM-Sal group and the difference was statistically significant compared to other groups.

Conclusion: CM-Sal derived from MSCs culture elicited significant histopathological improvement in LPS induced neuroinflammation which could be used as new therapeutic modality.

Keywords: Mesenchymal stem cells, Conditioned medium, Salidroside, Neuroinflammation

Accepted for publication December 7, 2016, Published online May 30, 2017

Correspondence to Zeinab M. El Maadawi

Department of Histology and Cell Biology, Faculty of Medicine, Cairo University, Al-Manial 11562, Egypt

Tel: +2-023194656, Fax: +2-023687673

E-mail: zeinab.elmaadawi@kasralainy.edu.eg

(a) This is an open-access article distributed under the terms of the Creative Commons Attribution Non-Commercial License (http://creativecommons.org/ licenses/by-nc/4.0/), which permits unrestricted non-commercial use, distribution, and reproduction in any medium, provided the original work is properly cited.

Copyright (C) 2017 by the Korean Society for Stem Cells Research

\section{Introduction}

Inflammation is implicated in the progressive nature of neurodegenerative diseases such as Alzheimer's disease $(\mathrm{AD})$ which is characterized by progressive cognitive dysfunction and dementia caused by inflammation and neuronal loss particularly in the forebrain (1). Mesenchymal stem cells (MSCs) are considered as an ideal candidate for cellular therapy and promising preclinical studies using MSCs transplantation have been reported in various animal models of $\mathrm{AD}$ (2). However, MSCs do not persist 
within the host injured tissue and have a limited ability to replace lost cells $(3,4)$. Meanwhile, it has been reported (5) that the therapeutic effects of MSCs are largely attributed to their paracrine-mediated actions through release of trophic factors. More recently, the concept of using MSCs conditioned medium (CM), rather than MSCs transplantation, has been widely adopted in regenerative medicine (6). MSCs derived CM contains secreted growth factors that are referred to as secretome, microvesicles, or exosomes (7).

Salidroside (p-hydroxyphenethyl- $\beta$-D-glucoside, Sal), a phenylpropanoid glycoside isolated from the Chinese plant Rhodiola rosea, has been widely used as a traditional herbal medicine that revealed potent anti-inflammatory and anti-oxidant effects (8). Previous studies showed that Sal could induce rat MSCs to differentiate into cholinergic neurons (9) and dopaminergic neurons (10). Concurrently, Sal has been reported to exert anti-inflammatory and neuroprotective effects on cultured murine microglial cells (1). Therefore, this study was planned to investigate the possible effect of CM derived from Sal-pretreated MSCs culture on a mouse model of neuroinflammation induced by lipopolysaccharide (LPS) $(11,12)$.

\section{Materials and Methods}

\section{Culture of bone marrow derived MSCs (13)}

MSCs were obtained from the bone marrow of femurs and tibias of adult male mice and were characterized as previously described (13). Bones were flushed with Dulbecco's Modified Eagle's medium (DMEM) (Sigma, USA) supplemented with $10 \%$ fetal bovine serum (FBS) (Sigma, USA). Bone marrow was slowly layered over Ficoll-Hypaque (Sigma, USA) and then centrifuged at $1200 \mathrm{rpm}$ for 30 minutes at room temperature. The opaque layer containing mononuclear cells was aspirated and re-suspended in complete culture medium (DMEM with 10\% FBS) supplemented with $1 \%$ penicillin-streptomycin (Sigma, USA). The medium was changed every other day and cells were passaged when reaching $90 \%$ confluence. Characterization of bone marrow derived MSCs was confirmed as reported before (6) based on their morphological criteria, plastic adherence and positive expression of CD90 and CD271 and negative expression of CD45.

\section{Preparation of MSCs conditioned medium $(6,14)$}

When reached $90 \%$ confluence, MSCs passage 2 were washed 3 times with phosphate-buffered saline (PBS) and seeded at $1 \times 10^{4}$ cells $/ \mathrm{ml}$ in 24-well plates containing serum-free DMEM (14). Each 24-well plate was divided equally (12 wells) into CM and CM-Sal in which the latter was exposed to $100 \mu \mathrm{g} / \mathrm{mL}$ salidroside (purity $\geq 95 \%$ (LC/MS-ELSD), Sigma-Aldrich, MO, USA) for $48 \mathrm{~h}$ of culture (10). Then CM and CM-Sal were collected separately, and centrifuged at $1500 \mathrm{rpm}$ for $5 \mathrm{~min}$. The supernatants were re-centrifuged at $3000 \mathrm{rpm}$ for $3 \mathrm{~min}$, filtered and the second supernatants were collected and designated as CM and CM-Sal respectively. Serum-free DMEM, similarly centrifuged and filtered, was used as control medium.

\section{Experimental design}

The study included 50 adult male mice (25 30 gm) that were housed in hygienic conditions at the Animal House of the Faculty of Medicine, Cairo University. They were allowed free access to food and water and were maintained on 12 hours light cycle. All procedures were held according to the guidelines of the Animal Research Committee of Cairo University. Animals were randomly divided into 5 groups (10 mice each) as follows:

Group I (Normal control): Received a single intraperitoneal (IP) injection of $0.5 \mathrm{ml}$ PBS followed, 24 hours later, by intranasal injection of $50 \mu 1$ of PBS twice daily for 4 days.

Group II (LPS): Lipopolysaccharide (LPS) (0127:B8, Cat. no. 63H-4010; Sigma-Aldrich, MO, USA) was injected in a single IP dose of $0.8 \mathrm{mg} / \mathrm{kg}$ dissolved in 0.5 $\mathrm{ml}$ PBS (12) followed, 24 hours later, by intranasal injection of $50 \mu 1$ of PBS twice daily for 4 days.

Group III (LPS-DMEM): LPS was injected as described in group II. Twenty four hours later, animals were subjected to intranasal injection of $50 \mu 1$ of filtered serum-free DMEM twice daily for 4 days.

Group IV (LPS-CM): LPS was injected as described in group II. Twenty four hours later, animals were subjected to intranasal injection of $50 \mu 1$ of $\mathrm{CM}$ twice daily for 4 days (14).

Group V (LPS-CM-Sal): LPS was injected as described in group II. Twenty four hours later, animals were subjected to intranasal injection of $50 \mu 1$ of CM-Sal twice daily for 4 days.

All animals were sacrificed at day 6 and the brain of each animal was fixed immediately in $10 \%$ formol saline and processed into paraffin blocks. Serial coronal sections (5 $\mu \mathrm{m}$ thickness) of the frontal cerebral cortex were prepared for further histological and immunohistochemical staining.

\section{Histological study}

Paraffin cerebral sections obtained from all groups were stained with Hematoxylin and Eosin (H\&E) (15) for histo- 
pathological evaluation.

\section{Immunohistochemistry study}

Rabbit polyclonal primary antibodies were purchased from abcam, Cambridge, MA, USA. Heat mediated-antigen retrieval was recommended according to the manufacturer's instructions using citric acid $\mathrm{pH} 6$ for 10 minutes. Paraffin sections were subjected to immunostaining with the following primary antibodies; cysteine aspartic protease-3 (caspase 3) (1:50) as an apoptotic marker (16), glial fibrillary acidic protein GFAP (1:500) for detection of astrocytes (17) and CD 68 (1:200) for detection of active microglia (18). For each primary antibody, cells were incubated with the primary antibody overnight at $4^{\circ} \mathrm{C}$. Secondary antibody detection system (universal Dako labeled Streptavidin-Biotin 2 system, Horseradish Peroxidase, LSAB2 System, HRP) (Dako, Carpinteria, CA, USA) was used for visualization followed by nuclear counter staining with Mayer's hematoxylin. All used primary antibodies demonstrated cytoplasmic reaction. Negative control was incorporated in each run through which no primary antibody was applied. Slides were examined by Olympus light microscope with digital camera (BX51; Olympus, Tokyo,
Japan).

\section{Quantitative morphometric study and statistical analysis}

The mean area percent of positive immune-staining for caspase 3, GFAP and CD68 were measured in 10 randomly selected non-overlapping high power fields (HPF) from 5 slides obtained from each specimen using $\times 40$ objective lens. All measurements were done using the binary mode of Leica Qwin 500 LTD image analysis computer system (Cambridge, UK). The results were expressed as mean \pm SD and significant differences were evaluated using one way analysis of variance (ANOVA) test. $\mathrm{p}$ value $<0.05$ was considered to be statistically significant (19).

\section{Results}

\section{Histological and immunohistochemical results}

$H \& E$ results: Examination of $H \& E$ stained sections of group I (normal control) revealed normal cerebral architecture in which multiple neurons with large pale vesicular nuclei were identified among glial cells with small dark nuclei (Fig. 1A). Histological findings of group II (LPS) and group III (LPS- DMEM) were similar in which
A

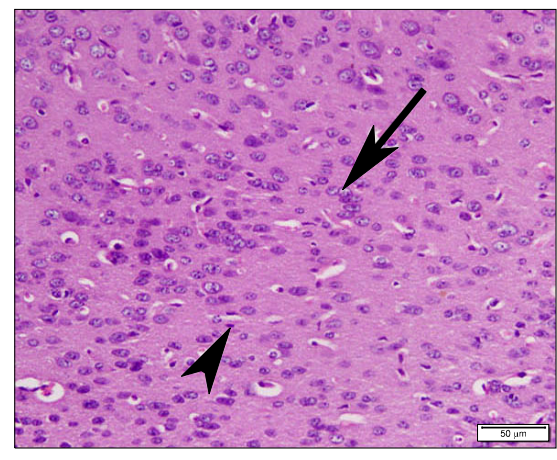

D

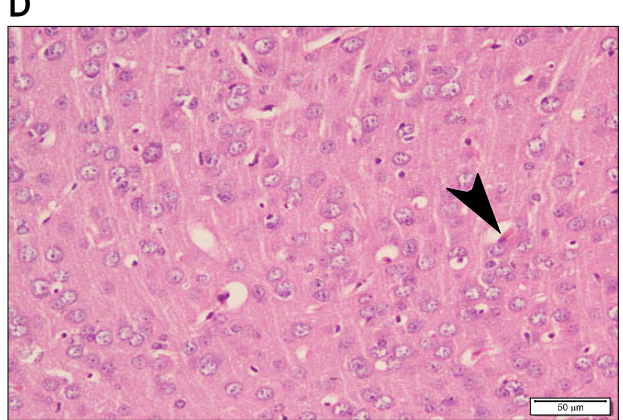

B

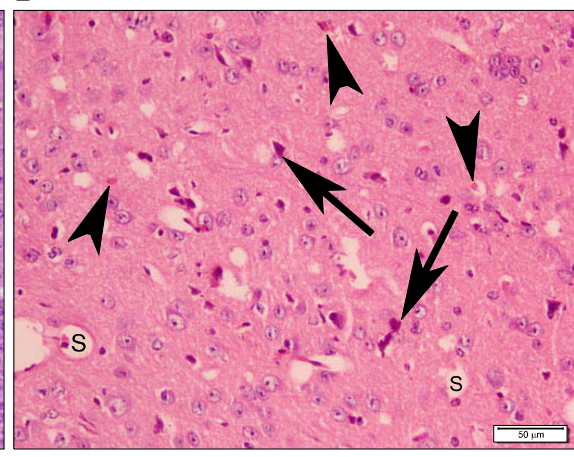

E
C
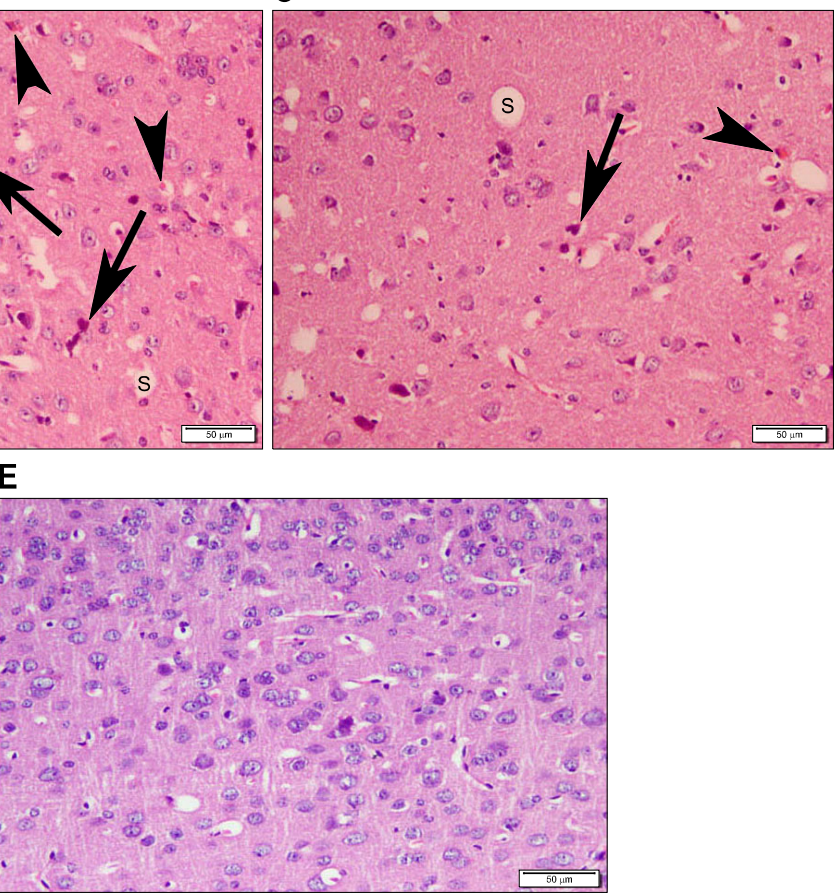

Fig. 1. Photomicrograph of mice frontal cortex (A) Group I (normal control) showing normal cerebral architecture with multiple neurons exhibiting large pale vesicular nuclei (arrow) among glial cells that could be identified by their small dark nuclei (arrow head). (B) Group II (LPS) and (C) Group III (LPS- DMEM) revealing multiple deformed neurons with dark shrunken nuclei (arrow) together with small acidophilic masses (arrow head) with marginated nuclei and multiple empty spaces (S) (D) Group IV (LPS-CM) and (E) Group V (LPS-CM-Sal) demonstrating preserved cerebral architecture with apparently less dark nuclei and very few acidophilic masses (arrow head) $(\mathrm{H} \& \mathrm{E}, \times 200)$. 
multiple deformed neurons with dark shrunken nuclei were demonstrated together with small acidophilic masses with marginated nuclei and multiple empty spaces (Fig. 1B and 1C). In group IV (LPS-CM) and group V (LPS-CM-Sal), cerebral architecture was preserved with apparently less dark nuclei and very few acidophilic masses (Fig. 1D and 1E).

Immunohistochemical results: In order to further evaluate the histopathological changes of all studied groups, immunohistochemistry was done to examine Caspase 3 immunoreaction for apoptosis as well as the immunoreactivity of GFAP for astrocytes and CD68 for activated microglia, both are recorded as two distinctive cellular markers in neurodegenerative disorders.

Caspase 3 immunohistochemistry: Examination of caspase 3 stained sections of group I (normal control) revealed scanty positive cytoplasmic staining in very few cells (Fig. 2A) while more positive cytoplasmic staining was apparently noticed in group II (LPS) and group III (LPS-DMEM) (Fig. 2B and 2C). Examination of caspase3 stained sections of group IV (LPS-CM) and group $\mathrm{V}$ (LPS-CM-Sal) demonstrated apparent decrease of im- munoreaction (Fig. 2D and 2E) compared to group II and group III.

GFAP immunohistochemistry: Examination of GFAP stained sections of group I (normal control) revealed typical cytoplasmic staining of astrocytes that demonstrated multiple branched cytoplasmic processes (Fig. 3A). Obvious increase in GFAP immunoreaction was noticed in group II (LPS) and group III (LPS-DMEM) (Fig. 3B and 3C) compared to group I while apparent less immunoreaction was recorded in group IV (LPS-CM) and group $V$ (LPS-CM-Sal) (Fig. 3D and 3E).

CD68 immunohistochemistry results: Examination of CD68 immunostained sections of group I (normal control) showed very few positive stained microglia (Fig. 4A). Meanwhile, more CD68 immunostaining was apparently noticed in group II (LPS) and group III (LPS-CM) in which many positively stained microglia exhibited rounded or oval shape (Fig. 4B and 4C). Apparent decrease in CD68 positive microglia was recorded in group IV (LPS-CM) and group V (LPS-CM-Sal) (Fig. 4D and 4E).
A

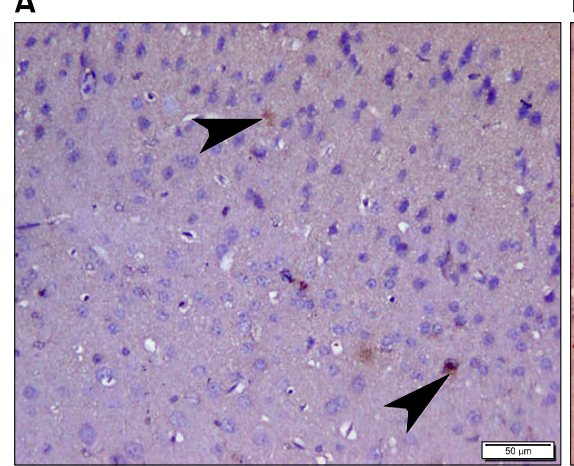

D

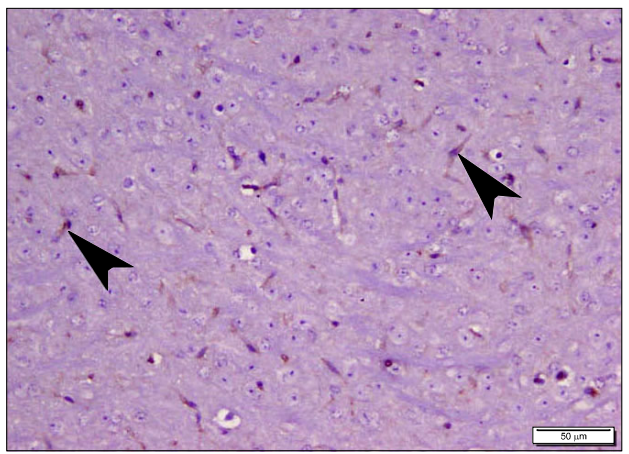

B

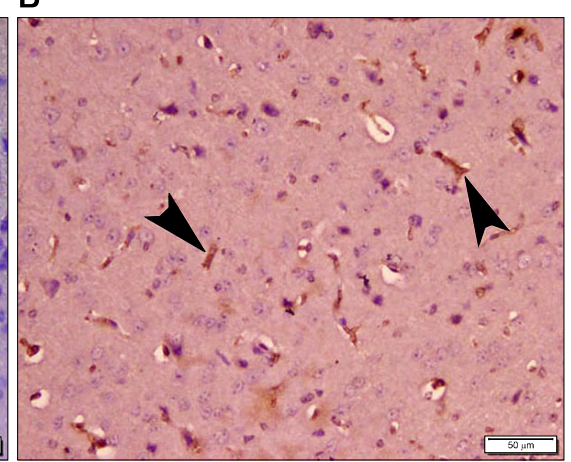

E

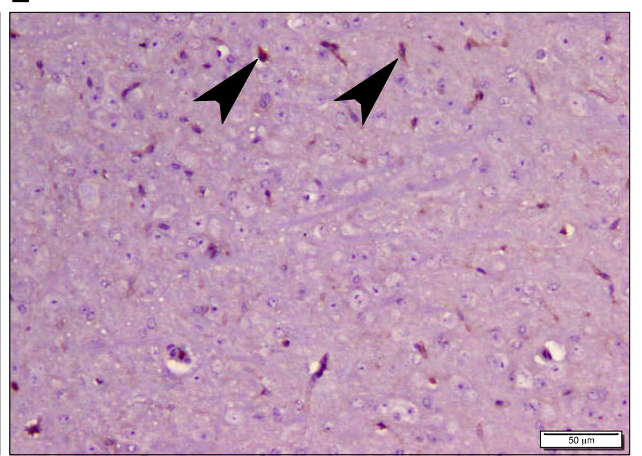

Fig. 2. Photomicrograph of mice frontal cortex (A) Group I (normal control) showing scanty positive caspase 3 cytoplasmic staining (arrow head) in very few cells. (B) Group II (LPS) and (C) Group III (LPS- DMEM) revealing more positive caspase 3 cytoplasmic staining (arrow head). (D) Group IV (LPS-CM) and (E) Group V (LPS-CM-Sal) demonstrating apparent decrease of caspase 3 immunoreaction (arrow head) (Caspase 3 immunohistochemistry, $\times 200$ ). 
A

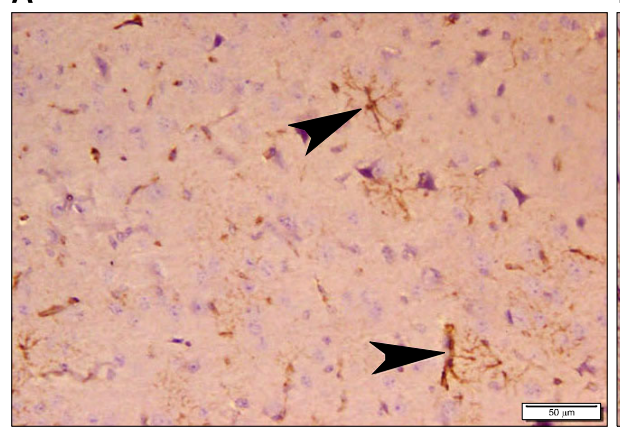

D

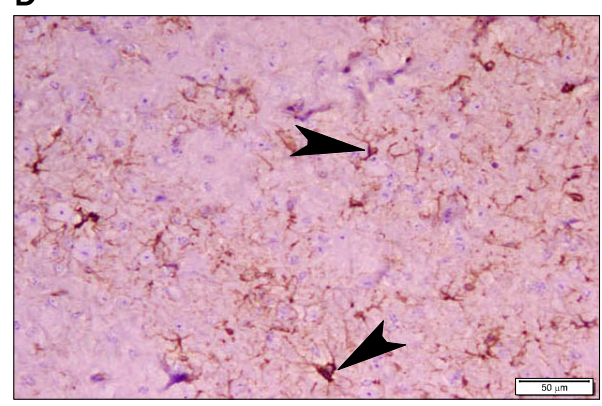

B

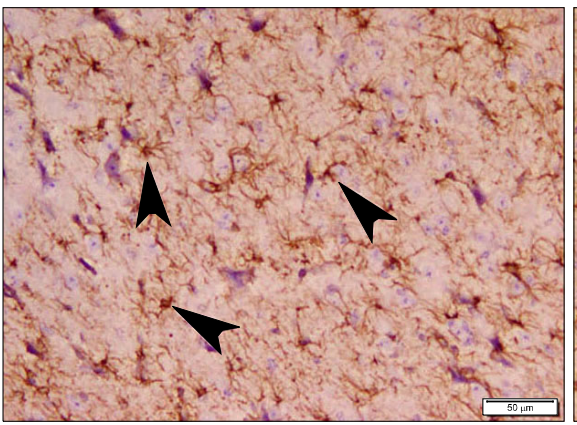

E
C

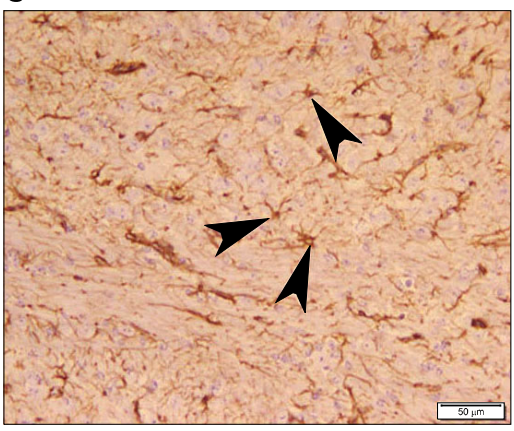

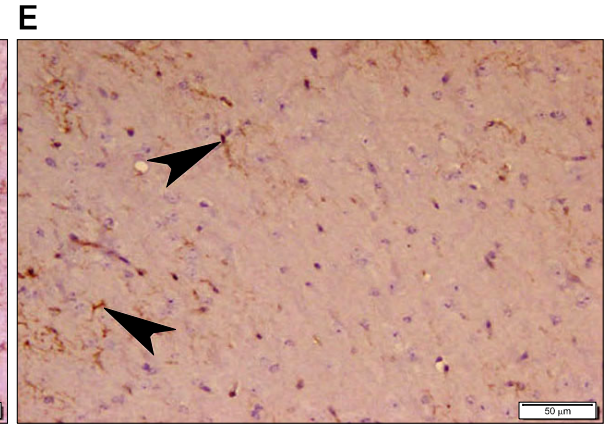

Fig. 3. Photomicrograph of mice frontal cortex (A) Group I (normal control) showing typical positive GFAP cytoplasmic staining of astrocytes (arrow head) that demonstrate multiple branched cytoplasmic processes. (B) Group II (LPS) and (C) Group III (LPS- DMEM) revealing obvious increase in GFAP immunoreaction of astrocytes (arrow head). (D) Group IV (LPS-CM) and (E) Group V (LPS-CM-Sal) demonstrating apparent less GFAP immunoreaction of astrocytes (arrow head). (GFAP immunohistochemistry, $\times 200$ ).

A

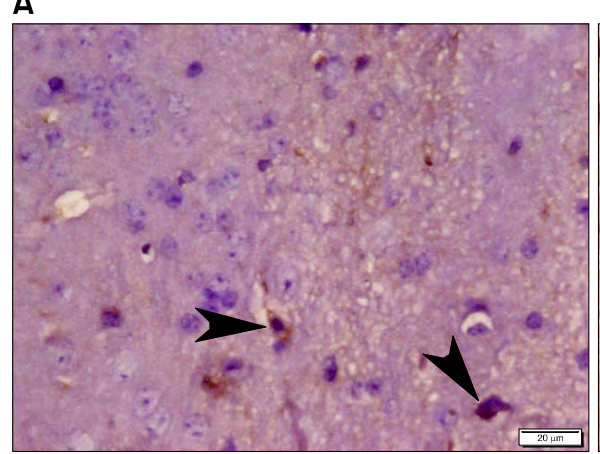

D
B

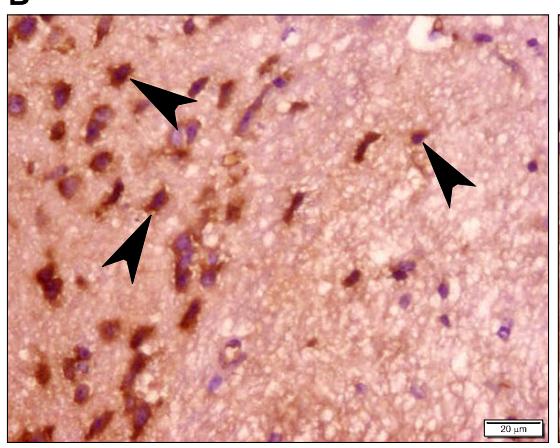

C

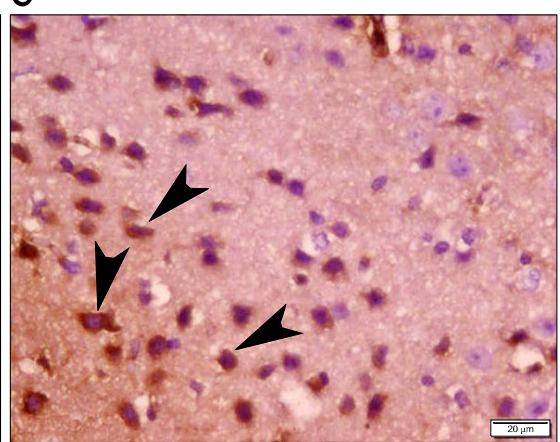

E
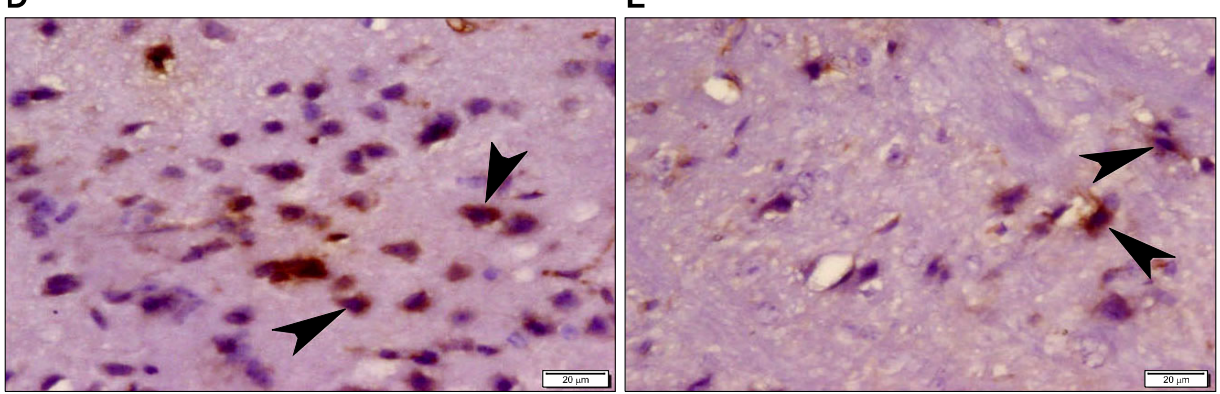

Fig. 4. Photomicrograph of mice frontal cortex (A) Group I (normal control) showing very few CD68 positive microglia (arrow head) (A). (B) Group II (LPS) and (C) Group III (LPS-DMEM) revealing more CD68 positive microglia (arrow head) that exhibited rounded or oval shape. (D) Group IV (LPS-CM) and (E) Group V (LPS-CM-Sal) demonstrating apparent decrease in CD68 positive microglia (arrow head). (CD68 immunohistochemistry, $\times 400)$. 
Table 1. Mean area percent \pm SD of caspase3, GFAP and CD68 immuno-expression in control and experimental groups

\begin{tabular}{|c|c|c|c|}
\hline & Caspase3 & GFAP & CD68 \\
\hline Group I (Normal control) & $0.73 \pm 0.18$ & $24.41 \pm 0.62$ & $2.47 \pm 0.31$ \\
\hline Group II (LPS) & $11.19 \pm 0.31 *$ & $41.90 \pm 0.80 *$ & $16.09 \pm 0.78^{*}$ \\
\hline Group III (LPS-DMEM) & $11.17 \pm 0.45^{*}$ & $42.25 \pm 0.99 *$ & $16.09 \pm 0.18^{*}$ \\
\hline Group IV (LPS-CM) & $6.73 \pm 0.39 *, \#, \Omega$ & $35.33 \pm 0.83 *, \#, \Omega$ & $9.15 \pm 0.41^{*}, \#, \Omega$ \\
\hline Group V (LPS-CM-Sal) & $4.08 \pm 0.62^{*, \#, \Omega, \Phi}$ & $30.29 \pm 0.46^{*, \#, \Omega, \Phi}$ & $5.26 \pm 0.33^{*, \#, \Omega, \uparrow}$ \\
\hline
\end{tabular}

$* \mathrm{p}<0.05$ : significant as compared to Group I.

${ }^{\#} \mathrm{p}<0.05$ : significant as compared to Group II.

${ }^{\Omega} \mathrm{p}<0.05$ : significant as compared to Group III.

${ }_{\mathrm{p}}<0.05$ : significant as compared to Group IV.

\section{Quantitative morphometric results}

The mean area percent of caspase 3, GFAP and CD68 immunoreaction was significantly increased in both LPS and LPS-DMEM groups compared to normal control, LPS-CM and LPS-CM-Sal groups. No significant difference was recorded between LPS and LPS-DMEM groups. Meanwhile, significant increase in the mean area percent of caspase 3, GFAP and CD68 immunoreaction was observed comparing either LPS-CM group or LPS-CM-Sal group to normal control group. However, the mean area percent of caspase 3, GFAP and CD68 in LPS-CM group was significantly higher than that of LPS-CM-Sal group (Table 1).

\section{Discussion}

LPS-induced neuronal injury represents one of the current standard models for studying neuro-inflammation both in vivo and in vitro (11). Local and chronic neuro-inflammation is a constant finding of neurodegenerative disorders such as $\mathrm{AD}$ and is characterized by activated microglia and astrocytes (20). In this study, the frontal cerebral cortex was considered as the mean area of interest for examining the histopathological findings of LPS induced neuroinflammation as it was reported that the histopathogenesis that features $\mathrm{AD}$ appears first in the frontal cortex then spreads over the entire cortical region (1).

Examination of H\&E stained sections in LPS group revealed small acidophilic masses with small dark nuclei and multiple deformed neurons together with multiple empty spaces that might be indicative of apoptosis and neuronal degeneration (11). In this study, features of neuronal degeneration and apoptosis detected in H\&E stained sections of LPS group were reinforced by the increase in the mean area percent of caspase 3 immunohistochemistry and were in agreement with other research (21). It was reported that oxidative stress is a common mechanism con- tributing to neuronal damage and apoptosis in neuroinflammatory disorders (22).

Additionally, glial cells increase secondary to the inflammatory process (11) and substantial evidence now demonstrates that astrocytes (20) and microglia (23) play critical roles in the pathogenesis of neuroinflammatory disorders including $\mathrm{AD}$.

Astrocytes express GFAP which is a principal intermediate filament in mature astrocytes and a reliable marker for their identification (17). In the current study, the mean area percent of GFAP in LPS group was significantly higher than normal control which has been described as astrogliosis in accordance to other reports $(24,25)$. Activated astrocytes release proinflammatory cytokines that promote recruitment of monocytes/microglia to the injured area (26). The interactions between dysfunctional astrocytes and neighboring neurons induces a vicious circle through which more neuronal injury occurs (20).

Regarding microglia, CD68 antibody is known to demonstrate microglial phagocytic activity as it labels lysosomal and endosomal transmembrane $(18,27)$. The mean area percent of CD68 positive microglia was significantly high in LPS group of the current study compared to normal control. In this study, CD 68 positive microglia exhibited rounded or oval shape without obvious branching processes, a finding which was previously reported as an indicative of microglial activity (6). Active microglia are known to exert neuroprotective effects through the release of neurotrophic factors and removal of cellular debris by phagocytosis $(28,29)$.

On the other hand, activated microglia produce large amounts of reactive oxygen species (ROS), nitric oxide (NO), and pro-inflammatory cytokines such as tumor necrosis factor (TNF-alpha), interleukin-1beta (IL-1beta), and interleukin-6 (IL-6), therefore they aggravate neuronal injury $(30,31)$. Moreover, others postulated that active microglia can exacerbate tissue damage via phagocytosis 
of functioning neurons and synapses (32).

In fact, two distinct subpopulations of microglia have been previously identified as M1 (proinflammatory) and M2 (anti-inflammatory) and the balance between the two types critically determines the pathophysiology of neurodegenerative disorders (14). Recently, microglial immunophenotyping in human postmortem brain specimens revealed that CD68 positive microglia, which are responsible for clearance of cellular debris, are positively associated with $\mathrm{AD}$ histopathological findings as well as impaired cognitive function (18).

In this study, the use of CM derived from MSCs, rather than transplantation of MSCs, was adopted in LPS induced neuroinflammation as $\mathrm{CM}$ provides easily handled, cell-free therapeutic modality that does not necessitate donor-recipient matching (33). It was suggested that MSCs culture conditions could influence their secretome and the constituents of secreted growth factors (6). Meanwhile, Sal, which is one of the major compounds of Rhodiola rosea, retains significant neuroprotective and antioxidative effects (34). Hence, the current study investigated the possible histopathological effect of Sal-pretreated CM derived from bone marrow MSCs on LPS induced neuronal injury.

In the current study, intranasal administration of CM and CM-Sal was experimented in a mouse model of LPS induced neuroinflammation. Intranasal therapy has been widely used for neurodegenerative disorders being less invasive and more efficient compared to intravenous route $(14,35)$.

It is worth mentioning that, in this study, serum was eliminated from CM, CM-Sal and DMEM control medium because of its poorly defined composition according to previous report (6).

This study revealed that treatment with $\mathrm{CM}$ and CM-Sal resulted in attenuation of neuronal apoptosis and diminished astrocyte and microglia reactivity as evidenced by significant decrease in the mean area percent of caspase 3, GFAP and CD68, respectively, in comparison to the untreated LPS group.

The anti-inflammatory effect of intranasal administration of CM derived from mouse bone marrow MSCs reported in this study is in agreement with a previous study (14) that examined the effect of CM derived from human bone marrow MSCs and stem cells from human exfoliated deciduous teeth (SHEDs) in a mouse model of $\mathrm{AD}$ that was induced by intracerebroventricular injection of A beta peptide. It can be explained by the ability of $\mathrm{CM}$ to inhibit the generation of both 3-nitrotyrosine (3-NT) and inducible nitric oxide synthase (iNOS) (14).

Alternatively, CM derived from rat bone marrow MSCs was found to provide protection against neuronal apoptosis in vitro (6). In addition, nerve growth factor (NGF) and brain-derived neurotrophic factor (BDNF), which are known to reduce reactive astrogliosis $(36,37)$, were identified in substantial amounts in CM derived from rat bone marrow MSCs (6).

Concomitantly, the improvement of histopathological findings was more significant in CM-Sal group compared to CM group. To the best of available knowledge, the current study is the first to examine the therapeutic effect of CM-Sal on LPS induced neuoinflammation in vivo.

Salidroside was found to promote the secretion of BDNF and NGF and to increase the expression of their mRNAs in rat bone marrow MSCs culture (10) which could explain the accelerated improvement noticed in $\mathrm{CM}$-Sal group compared to $\mathrm{CM}$ group.

In addition, it was previously documented that Rhodiola rosea constituents, including Sal, strongly inhibit NO production and the expression of iNOS, the key enzyme for NO production, in LPS-stimulated microglial cells in vitro (1), therefore favoring antioxidant and anti-inflammatory effects. Consequently this can explain the marked decrease in the mean area percent of CD68 positive active microglia reported in CM-Sal group of the current study.

Interestingly, salidroside, cultured for 12 72 hours with mouse bone marrow MSCs in neuronal induction medium, was reported to induce neuronal differentiation through down regulation of Notch signaling and up regulation of BMP signaling pathways (8) which might be accompanied by release of more neurotrophic factors in MSCs differentiated medium. Further investigations targeting the molecular expression of Notch and BMP signaling pathways (8) are needed to explain the exact mechanisms through which CM-Sal elucidated improvement which is considered one imitation in the current study.

Several in vitro studies reinforced Sal antioxidant and anti-inflammatory effects. Sal diminishes A beta-induced oxidative damage on rat neuronal PC12 cells and on SH-SY5Y human neuroblastoma cells (38). MADP, a salidroside analog, was found to protect cultured rat hippocampal neurons from glutamate induced apopotosis (39). In addition, Sal was able to improve the viability of primary rat astrocyte culture and to attenuate oxidative stress by diminishing spontaneous ROS (40) which might explain the reduction in reactive astrogliosis reported in the current study.

\section{Conclusion}

The administration of CM or CM-Sal derived from 
MSCs culture revealed significant histopathological improvement in LPS induced neuroinflammation. The accentuated effect of CM-Sal firstly reported in this study could be attributed to the summative influence of both $\mathrm{CM}$ and Sal on suppression of inflammation, inhibition of apoptosis and neuroprotection. Therefore, intranasal administration of CM-Sal is assumed to be an alternative, effective, cell-free therapy for histopathological repair of neuroinflammation which needs to be functionally evaluated in different animal models. Further safety assessment is warranted before any clinical trials.

\section{Potential conflict of interest}

The authors have no conflicting financial interest.

\section{References}

1. Lee Y, Jung JC, Jang S, Kim J, Ali Z, Khan IA, Oh S. Anti-inflammatory and neuroprotective effects of constituents isolated from rhodiola rosea. Evid Based Complement Alternat Med 2013 doi: 10.1155/2013/514049

2. Li M, Guo K, Ikehara S. Stem cell treatment for Alzheimer's disease. Int J Mol Sci 2014;15:19226-19238

3. Lee JK, Jin HK, Endo S, Schuchman EH, Carter JE, Bae JS. Intracerebral transplantation of bone marrow-derived mesenchymal stem cells reduces amyloid-beta deposition and rescues memory deficits in Alzheimer's disease mice by modulation of immune responses. Stem Cells 2010;28: 329-343

4. Yang H, Xie Z, Wei L, Yang H, Yang S, Zhu Z, Wang P, Zhao C, Bi J. Human umbilical cord mesenchymal stem cell-derived neuron-like cells rescue memory deficits and reduce amyloid-beta deposition in an $\mathrm{A} \beta \mathrm{PP} / \mathrm{PS} 1$ transgenic mouse model. Stem Cell Res Ther 2013;4:76

5. Uccelli A, Laroni A, Freedman MS. Mesenchymal stem cells for the treatment of multiple sclerosis and other neurological diseases. Lancet Neurol 2011;10:649-656

6. Cantinieaux D, Quertainmont R, Blacher S, Rossi L, Wanet T, Noël A, Brook G, Schoenen J, Franzen R. Conditioned medium from bone marrow-derived mesenchymal stem cells improves recovery after spinal cord injury in rats: an original strategy to avoid cell transplantation. PLoS One 2013;8:e69515

7. Kim HO, Choi SM, Kim HS. Mesenchymal stem cell-derived secretome and microvesicles as a cell-free therapeutics for neurodegenerative disorders. Tissue Eng Regen Med 2013;10:93-101

8. Zhao HB, Qi SN, Dong JZ, Ha XQ, Li XY, Zhang QW, Yang YS, Bai J, Zhao L. Salidroside induces neuronal differentiation of mouse mesenchymal stem cells through Notch and BMP signaling pathways. Food Chem Toxicol 2014;71:60-67

9. Zhang M, Zhao H, Li Z, Yang Y, Wen Y, Dong J, Zhang Q, Ge B. Effect of salidroside on rat bone marrow mesen- chymal stem cells differentiation into cholinergic nerve cells. Zhongguo Xiu Fu Chong Jian Wai Ke Za Zhi 2012; 26:158-165

10. Zhao HB, Ma H, Ha XQ, Zheng P, Li XY, Zhang M, Dong JZ, Yang YS. Salidroside induces rat mesenchymal stem cells to differentiate into dopaminergic neurons. Cell Biol Int 2014;38:462-471

11. Nazem A, Sankowski R, Bacher M, Al-Abed Y. Rodent models of neuroinflammation for Alzheimer's disease. J Neuroinflammation 2015;12:74-88

12. Khairallah MI, Kassem LA, Yassin NA, Gamal el Din MA, Zekri M, Attia M. Activation of migration of endogenous stem cells by erythropoietin as potential rescue for neurodegenerative diseases. Brain Res Bull 2016;121:148-157

13. Alhadlaq A, Mao JJ. Mesenchymal stem cells: isolation and therapeutics. Stem Cells Dev 2004;13:436-448

14. Mita T, Furukawa-Hibi Y, Takeuchi H, Hattori H, Yamada K, Hibi H, Ueda M, Yamamoto A. Conditioned medium from the stem cells of human dental pulp improves cognitive function in a mouse model of Alzheimer's disease. Behav Brain Res 2015;293:189-197

15. Kiernan JA. Histological and histochemical methods: theory and practice. 3rd ed. London, New York \& New Delhi: Arnold publisher; 2001. 111-162

16. Khan MS, Ali T, Kim MW, Jo MH, Jo MG, Badshah $H$, Kim MO. Anthocyanins protect against LPS-induced oxidative stress-mediated neuroinflammation and neurodegeneration in the adult mouse cortex. Neurochem Int 2016;100: $1-10$

17. Korolainen MA, Auriola S, Nyman TA, Alafuzoff I, Pirttilä T. Proteomic analysis of glial fibrillary acidic protein in Alzheimer's disease and aging brain. Neurobiol Dis 2005; 20:858-870

18. Minett T, Classey J, Matthews FE, Fahrenhold M, Taga M, Brayne C, Ince PG, Nicoll JA, Boche D; MRC CFAS. Microglial immunophenotype in dementia with Alzheimer's pathology. J Neuroinflammation 2016;13:135

19. Emsley R, Dunn G, White IR. Mediation and moderation of treatment effects in randomised controlled trials of complex interventions. Stat Methods Med Res 2010;19:237-270

20. Avila-Muñoz E, Arias C. When astrocytes become harmful: functional and inflammatory responses that contribute to Alzheimer's disease. Ageing Res Rev 2014;18:29-40

21. Bassiony HS, Zickri MB, Metwally HG, Elsherif HA, Alghandour SM, Sakr W. Comparative histological study on the therapeutic effect of green tea and stem cells in Alzheimer's disease complicating experimentally induced diabetes. Int J Stem Cells 2015;8:181-190

22. Cassano T, Pace L, Bedse G, Lavecchia AM, De Marco F, Gaetani S, Serviddio G. Glutamate and mitochondria: two prominent players in the oxidative stress-induced neurodegeneration. Curr Alzheimer Res 2016;13:185-197

23. ElAli A, Rivest S. Microglia in Alzheimer's disease: A multifaceted relationship. Brain Behav Immun 2016;55:138-150

24. Fu HQ, Yang T, Xiao W, Fan L, Wu Y, Terrando N, Wang TL. Prolonged neuroinflammation after lipopolysaccharide 
exposure in aged rats. PLoS One 2014;9:e106331.

25. Kamphuis W, Middeldorp J, Kooijman L, Sluijs JA, Kooi EJ, Moeton M, Freriks M, Mizee MR, Hol EM. Glial fibrillary acidic protein isoform expression in plaque related astrogliosis in Alzheimer's disease. Neurobiol Aging 2014;35: 492-510

26. Lee KM, MacLean AG. New advances on glial activation in health and disease. World J Virol 2015;4:42-55.

27. Rabinowitz SS, Gordon S. Macrosialin, a macrophage-restricted membrane sialoprotein differentially glycosylated in response to inflammatory stimuli. J Exp Med 1991;174: 827-836

28. Neumann J, Gunzer M, Gutzeit HO, Ullrich O, Reymann KG, Dinkel K. Microglia provide neuroprotection after ischemia. FASEB J 2006;20:714-716

29. Boido M, Garbossa D, Fontanella M, Ducati A, Vercelli A. Mesenchymal stem cell transplantation reduces glial cyst and improves functional outcome after spinal cord compression. World Neurosurg 2014;81:183-190

30. González-Scarano F, Baltuch G. Microglia as mediators of inflammatory and degenerative diseases. Annu Rev Neurosci 1999;22:219-240

31. Minghetti L, Levi G. Microglia as effector cells in brain damage and repair: focus on prostanoids and nitric oxide. Prog Neurobiol 1998;54:99-125

32. Brown GC, Neher JJ. Microglial phagocytosis of live neurons. Nat Rev Neurosci 2014;15:209-216

33. Pawitan JA. Prospect of stem cell conditioned medium in regenerative medicine. Biomed Res Int 2014 doi: 10.1155/
2014/965849

34. Sun ZK, Yang HQ, Chen SD. Traditional Chinese medicine: a promising candidate for the treatment of Alzheimer's disease. Transl Neurodegener 2013;2:6

35. Thorne RG, Pronk GJ, Padmanabhan V, Frey WH 2nd. Delivery of insulin-like growth factor-I to the rat brain and spinal cord along olfactory and trigeminal pathways following intranasal administration. Neuroscience 2004;127:481496

36. Cirillo G, Bianco MR, Colangelo AM, Cavaliere C, Daniele de L, Zaccaro L, Alberghina L, Papa M. Reactive astrocytosis-induced perturbation of synaptic homeostasis is restored by nerve growth factor. Neurobiol Dis 2011;41:630639

37. Jain A, McKeon RJ, Brady-Kalnay SM, Bellamkonda RV. Sustained delivery of activated Rho GTPases and BDNF promotes axon growth in CSPG-rich regions following spinal cord injury. PLoS One 2011;6:e16135

38. Liu C, Yuan Y, Zhang M. Uncertainty analysis of displacement measurement with Imetrum Video Gauge. ISA Trans 2016;65:547-555

39. Xian H, Zhao J, Zheng Y, Wang M, Huang J, Wu B, Sun C, Yang Y. MADP, a salidroside analog, protects hippocampal neurons from glutamate induced apoptosis. Life Sci 2014;103:34-40

40. Zhao C, Zhu Z, Wang Y, Reiser G, Tang L. Protection of salidroside on primary astrocytes from cell death by attenuating oxidative stress. Chinese Herbal Medicines 2015;7: 303-309 